\title{
L'importance des études à résultats négatifs ou neutres pour l'évolution de la pratique clinique
}

\author{
par Lauren Bresee
}

$\int^{\prime}$ ai eu la chance de travailler avec plusieurs chercheurs débutants au cours de ma carrière, notamment des résidents en pharmacie, des étudiants diplômés et des pharmaciens. Épauler les tout nouveaux chercheurs est l'une de mes activités préférées : servir de mentor à des personnes intéressées par la recherche est très gratifiant. Durant mon travail avec eux, ils m’ont parlé de certaines des idées fausses associées à la recherche. Lune des plus répandues est qu'une étude n'ayant pas de résultats positifs est un échec et qu'il n'y a donc rien à en tirer.

En ce qui concerne le présent éditorial, une étude à résultats positifs se définit comme celle dans laquelle l'intervention a des résultats bénéfiques statistiquement significatifs par rapport au comparateur. Une étude à résultats négatifs se définit comme celle dans laquelle un préjudice statistiquement significatif a été découvert. Une étude à résultats neutres se définit quant à elle comme celle qui ne présente aucune différence statistiquement significative entre l'intervention et ce à quoi elle est comparée'. Lexigence pour toutes ces définitions est que le plan de l'étude doit être adéquatement conçu et observé : plus précisément, l'étude doit avoir la puissance nécessaire pour relever une différence statistique s'il en existe une, le comparateur employé doit être adéquat et les tests statistiques utilisés doivent convenir au plan de l'étude et aux données à analyser.

Un biais de publication fait référence à la probabilité que les études à résultats positifs soient plus à même d'être publiées que celles à résultats négatifs ou neutres ${ }^{2}$. Le problème du biais de publication est bien connu et se manifeste de différentes façons, notamment : s'abstenir délibérément de diffuser les études à résultats négatifs ou ne pas pouvoir publier des études à résultats négatifs ou neutres dans les revues avec comité de lecture, car elles sont rejetées avant ou après la révision par les pairs ${ }^{3}$. Il est difficile de quantifier la fréquence des causes du biais de publication lié aux études à résultats négatifs ou neutres. Cependant, la conviction qu'une étude ne sera pas publiée du fait de ses résultats contribue probablement au biais de publication faute de soumettre ces manuscrits aux revues avec comité de lecture ${ }^{2}$.

Mais pourquoi les études à résultats négatifs sont-elles importantes? En tant que fournisseurs de soins de santé, nous voulons instinctivement offrir à nos patients les technologies les plus efficaces pour leur permettre d'atteindre leurs objectifs. Nous devons aussi être réalistes quant aux ressources limitées en santé au Canada, une contrainte qui fait ressortir la nécessité d'offrir aux patients les interventions qui leur seront réellement bénéfiques. Les études à résultats neutres ou négatifs nous aident à identifier les traitements inefficaces ou préjudiciables et nous pouvons ainsi agir pour que nos patients ne reçoivent pas ces traitements. Pour cette raison, ces études nous sont indispensables, car elles nous aident à trouver les soins adéquats pour nos patients.

Alors, comment vaincre le scepticisme face aux études à résultats négatifs? Parmi les recommandations ci-dessous, les deux premières s'appliquent à toutes les recherches, mais elles sont particulièrement importantes lorsqu'on prend en compte les études à résultats négatifs ou neutres. Premièrement, les chercheurs doivent soigneusement planifier et mener leurs études afin de s'assurer de la validité des résultats. Si l'on arrive à un résultat négatif ou neutre, avoir réalisé correctement l'étude permet de garantir que les résultats sont valides et qu'ils ne sont pas la conséquence d'une erreur fatale, comme un échantillon de taille inadéquate. Deuxièmement, les chercheurs doivent aborder leurs travaux en faisant preuve d'équilibre personnel, c'est-à-dire que le chercheur n'accorde pas de préférences personnelles préconçues selon lesquelles une ou plusieurs des interventions puissent produire de meilleurs résultats qu'une autré ${ }^{4}$. Aborder un projet de recherche avec équilibre pourrait éviter l'idée préconçue que l'étude sera une réussite seulement si elle met au jour un avantage. Ainsi, avec un peu de chance, cette attitude encouragera le chercheur à reconnaître la valeur de tous les 
résultats de sa recherche, qu'ils soient positifs ou non, et l'intérêt de les diffuser. Cette avenue peut être particulièrement difficile lorsque le chercheur a un intérêt personnel vis-à-vis de l'intervention évaluée. Un exemple serait l'évaluation de l'efficacité d'un programme par l'équipe de recherche même qui l'a élaboré pour en faire profiter ses patients. S'ils découvrent que leur programme est inefficace, voire préjudiciable, les chercheurs pourraient hésiter à diffuser les résultats de l'étude. Or, il est impératif de faire connaitre ces résultats aux autres fournisseurs de soins de santé qui pourraient être en train d'offrir des programmes semblables à leurs patients; ils pourraient ainsi prendre une décision éclairée quant à la continuation d'un tel programme. La troisième recommandation semble peut-être évidente, mais les chercheurs doivent chercher à publier leurs études à résultats négatifs ou neutres. Les rédacteurs des revues scientifiques sont d'ailleurs de plus en plus conscients du risque de biais de publication et sont donc intéressés à publier des études à résultats négatifs ou neutres. Par exemple l'American Journal of Gastroenterology a publié un "numéro négatif " en novembre 2016 : tous les articles y apparaissant présentaient des résultats statistiquement négatifs ou neutres 5 . Le Journal canadien de la pharmacie hospitalière accueille volontiers la présentation d'études à résultats négatifs ou neutres. Enfin, nous devons sensibiliser tous les cliniciens à l'importance des études à résultats négatifs, car elles sont nécessaires à l'évolution de la pratique clinique. Il ne faut pas s'attendre à ce que chaque intervention soit bénéfique pour nos patients; nous avons donc besoin des études à résultats négatifs ou neutres pour nous aider à trouver les interventions qui seront les plus efficaces pour nos patients.

[Traduction par l'éditeur]

\section{References}

1. Axelson O. Negative and non-positive epidemiological studies. Int J Occup Med Environ Health. 2004;17(1):115-21.

2. Olson CM, Rennie D, Cook D, Dickersin K, Flanagin A, Hogan JW, et al. Publication bias in editorial decision making. JAMA. 2002;287(21):2825-8.

3. Dwan K, Altman DG, Arnaiz JA, Bloom J, Chan AW, Cronin E, et al. Systematic review of the empirical evidence of study publication bias and outcome reporting bias. PLoS One. 2008;3(8):e3081.

4. Cook C, Sheets C. Clinical equipoise and personal equipoise: two necessary ingredients for reducing bias in manual therapy trials. J Man Manip Ther. 2011;19(1):55-7.

5. The American Journal of Gastroenterology presents "The Negative Issue". Twentyfive negative studies that remind readers that "negative is positive" [communiqué de presse]. Bethesda (MD) : American College of Gastroenterology; 2016. Publié au : www.prnewswire.com/news-releases/the-american-journal-ofgastroenterology-presents-the-negative-issue-300356821.html. Consulté le 23 août 2017.

Lauren Bresee, B. Sc. (Pharm.), ACPR, M. Sc., Ph. D., est conseillère scientifique à l'Agence canadienne des médicaments et des technologies de la santé (ACMTS), professeure agrégée adjointe au Département des sciences de la santé communautaire au Cumming School of Medicine de I'Université de Calgary et membre de I'O'Brien Institute for Public Health de I'Université de Calgary. Elle est également rédactrice adjointe du Journal canadien de la pharmacie hospitalière.

Intérêts concurrents : Aucun déclaré.

Adresse de correspondance :

Dre Lauren Bresee

Agence canadienne des médicaments et des technologies de la santé 865, avenue Carling, bureau 600

Ottawa (Ontario) K1S 5 S8

Courriel : LaurenB@cadth.ca 